\title{
Efficient Space Dedication to Bus Rapid Transit and Light Rail Systems
}

\author{
H.-S. Jacob Tsao, San Jose State University \\ Agus Pratama, Workday, Inc.
}

\begin{abstract}
Dedicating two lanes and passenger platforms to transit typically requires taking the same space away from general use. This may affect public support. This paper proposes efficient geometric configurations for a two-dedicated-lane BRT or light rail system that requires a minimum amount of right-of-way along a busy commute arterial. In many current busy commute corridors, a significant part of the street median is underused or unused for traffic purposes. The efficiency is achieved mainly by using the street median between a left-turn lane and its counterpart located at the intersection on the other end of the same street section and slanting part of the two dedicated lanes with respect to the longitudinal direction of the street. Instead of the three-lane or even four-lane conversion required of the prevailing configurations, the proposed configurations require conversion of only two lanes from general use, even for a section equipped with passenger platforms.
\end{abstract}

\section{Introduction}

Public transportation is perhaps one of the few sustainable transportation solutions for urban or suburban areas. Most, if not all, cities have public transportation systems. However, relatively few provide rapid transit systems. An urban rail or light rail system is the classical and conventional transit system used in most developed countries as well as in some cities of emerging economies (New Delhi, Beijing, Shanghai, etc.) while bus rapid transit (BRT) is a relatively new mass-transit concept that has been adopted by both developed countries and emerging economies 
(Levinson et al. 2002; Jarzab et al. 2002; Diaz et al. 2004; Miller et al. 2006; Kittelson \& Associates et al. 2007). The operational concepts and geometric configurations proposed in this paper are applicable and beneficial for both BRT and light rail. For ease of discussion, we address BRT explicitly in the rest of the paper and provide geometric sketches and justification only for BRT operations.

To minimize travel time and its variability for BRT, traffic lanes together with spaces required for the concomitant passenger activities along a street median can be dedicated to form a dedicated transitway (Li et al. 2009). In addition, transit signal priority (TSP) and other technologies can be adopted to improve system performance. However, the current vehicular traffic of many cities is dominated by automobiles. Such cities include perhaps most U.S. cities, with few exceptions (New York City and Chicago), and many cities in other developed nations or emerging economies. Dedicating two lanes in the street median and the additional spaces needed for bus stops often requires taking the same space away from use by automobiles. In prevailing geometric designs for dedicated BRT systems, passenger activities at a bus stop are accommodated with either two physically-separate passenger platforms (one for each direction) or one dual-use platform. In either case, the width of the required space is approximately the width of two traffic lanes. This kind of lane conversion could lead to heavy congestion during peak commute hours unless parallel streets or even corridors have sufficient capacity to accommodate the redirected traffic. In addition, the possible low bus-traffic volume on such a dedicated transitway before the demand for bus services can be gradually built up could lead to the impression of space underutilization; such impression is sometimes referred to as the "empty-lane syndrome." Such possible congestion and syndrome could lead to strong motorist resentment against implementation of BRT on a dedicated transitway. An alternative to such lane conversion is right-ofway purchase, but the cost may be prohibitively high and land-owner resentment may be strong. These may be primary reasons why few such dedicated systems have been implemented in North America.

Phase I of the Viva BRT system, designed for the York region of Ontario, Canada, was opened in 2005, and its Phase II, featuring several dedicated transitways accommodated on the street median called "rapidways," is being implemented with a full funding commitment of $\$ 1.4$ billion Canadian by the Province of Ontario (York Region Rapid Transit Corporation 2012). Much of the required additional right-of-way was purchased. Although the Orange Line of LA Metro has recently been implemented almost entirely on exclusive lanes (except for several blocks 
near the western end at the Warner Center), these lanes occupy the abundant right-of-way of an abandoned railroad and were built as a new, stand-alone road (Callaghan and Vincent 2007). For wide acceptance of BRT implemented with such a dedicated transitway in developed nations, conversion of existing right-of-way without significant right-of-way acquisition may be necessary and, therefore, efficient dedication of right-of-way for transit use is a critical issue. This motivated our research into this issue.

Many BRTs with a dedicated transitway have been implemented in emerging economies, in a societal context where the vast majority of the population already relies on public transportation. Such BRT systems, if implemented appropriately, would improve transit services for the majority, and proposals for building such systems tended to receive popular support. For widespread implementations of such BRT systems in the U.S. or other nations where urban and suburban transportation systems have been primarily developed for and used by automobile traffic, the benefit to transit users must be sufficiently compelling for winning over car drivers, and the negative impact on the automobile traffic must be minimized. Simply put, in emerging economies, bus transit is already popular and BRT is only expected to make it better; in the U.S., however, a successful BRT system must make transit popular. This is particularly true at the initial stages of a U.S. implementation, before transit-oriented development (TOD) can begin. The success of BRTs with a dedicated transitway in the U.S. may hinge upon efficient lane dedication or conversion and TSP.

Also motivated by the fact that the right-of-way required for a conventional twodedicated-lane BRT along many busy corridors either does not exist or is too costly to acquire, we proposed a one-dedicated-lane two-way (dynamically reversible) BRT system (Tsao et al. 2009a, 2009b, 2009c). A set of detailed operating rules, including design rules for giving signal priority to BRT vehicles at intersections along the one dedicated lane, for performance optimization has been developed (Tsao et al. 2010).

This paper proposes efficient geometric configurations for a two-dedicated-lane BRT system that require a minimum amount of the precious right-of-way along a busy commute arterial provided with frequent protected left-turn lanes. The efficiency of right-of-way utilization achieved with the proposed configurations results from capitalizing on the widespread existence of right-of-way unused or underused for traffic purposes along many current busy commute corridors in the U.S. The space in between the through lanes of one direction and the through lanes of the 
other direction is often occupied by one left-turn lane (or more) in each of the two directions and the median in between. The median is often quite long and planted with trees or shrubs. Although the plants serve aesthetic and other purposes, such a median is typically unused or underused for traffic purposes. The authors most certainly do not advocate paving over such green spaces in a first attempt to gain the required space; rather, we consider it only as a last resort and as a final enabler. A main idea behind the efficient configurations to be proposed in this paper is to add the two dedicated lanes in a slanted fashion (with respect to the longitudinal direction of the street) so as to more fully utilize such a median for traffic purposes.

The efficiency gain across the entire length of a BRT system is achieved independently and additively through such fuller utilization for individual sections. Therefore, the proposed configurations offer the highest efficiency-gain potential if the corridor consists of long sections and is equipped with a left-turn lane at each end of each of its sections. Instead of the three-lane or even four-lane conversion required for the prevailing configurations, the proposed configurations require a right-of-way width equivalent to only two lanes, even for a section equipped with passenger platforms. (Such a BRT system is not operated entirely on dedicated space, however, because its traffic lanes intersect with cross-streets at grade.)

Conventional light rail or BRT systems, already implemented (e.g., Lane Transit District 2002; Carey 2006) or being planned (e.g., AC Transit 2012a \& 2012b), do not capitalize on such unused or underused right-of-way, and their designs typically require dedication of right-of-way equivalent to three or four traffic lanes, particularly for sections accommodating a bus stop. In many cases, the three to four lanes have been or are to be converted from general-use lanes. We capitalize on such unused or underused median space and propose several geometric configurations accordingly. With the conversion of left-turn lanes to passenger platforms (only) at selected sections, we propose bus-lane configurations that require conversion of only two lanes throughout the system, for sections with or without a bus stop. We also propose a geometric configuration that uses the unused or underused right-ofway even more efficiently and requires conversion of exactly two lanes throughout the system. In this configuration, the left-turn lanes are retained, and one passenger platform (used for both directions) is located between the two dedicated bus lanes and is accommodated with the unused or underused median space. However, this platform must be accessed through mid-block pedestrian cross-walks.

In addition to the prevailing concepts of a two-dedicated-lane BRT system, many BRT or light rail concepts have been proposed or implemented for operations 
in mixed traffic (e.g., Institute for Transportation and Development Policy 2009; Levinson et al. 2002; Jarzab et al. 2002; Diaz et al. 2004; Miller et al. 2006; Kittelson \& Associates et al. 2007). Together with these concepts, the configurations and operational concepts proposed in this paper and those proposed in our earlier work for a one-dedicated-lane two-way system hopefully constitute a more complete spectrum of implementation options, at least from the view point of dedicating right-of-way along street median. For more details about the two-dedicatedlane system proposed in this paper or about how the one-dedicated-lane system proposed previously can be easily expanded to two dedicated lanes, the reader is referred to Tsao et al. (2009a, 2010).

The remainder of this paper is organized as follows. We first point out the right-ofway currently unused or underused for traffic purposes in the median along many busy commute corridors. We next discuss the conventional geometric designs for a two-dedicated-lane BRT system and propose more efficient designs in three separate sections. Concluding remarks are then given, together with related research findings that could not be reported in this paper due to space limitation and with worthy subjects for future research.

\section{Unused or Underused Median Space in Right-of-way of Urban Corridor with Frequent Left-turn Lanes}

Although the right-of-way of an arterial serving a busy corridor may be wider at interactions with major cross streets, the total width of the right-of-way dedicated to the rest of the roadway of such a corridor changes only occasionally. In particular, the width of a section between two adjacent intersections equipped with one left-turn lane for each (but opposite) direction typically remains constant. When compared to the length of such a section, a typical left-turn lane is rather short. On many arterials serving a busy corridor, a significant amount of median space exists along the roadway between two such adjacent intersections, and such median space is not useful for facilitating the through traffic on a conventional roadway. As a result, such median space is typically planted with trees or shrubs or is used for left-turning convenience into store parking lots. We, therefore, refer to such median space as "unused median space" or "underused median space." For discussion convenience, we use the former in the remainder of this paper. Figure 1 is a geometric sketch for such unused median space. This seven-lane configuration is used mainly to illustrate the existence of such unused space. It will be used later to illustrate how two general-purpose lanes can be converted efficiently to accom- 
modate two dedicated BRT lanes, without requiring any additional right-of-way. (This efficient conversion is illustrated in Figure 3.)

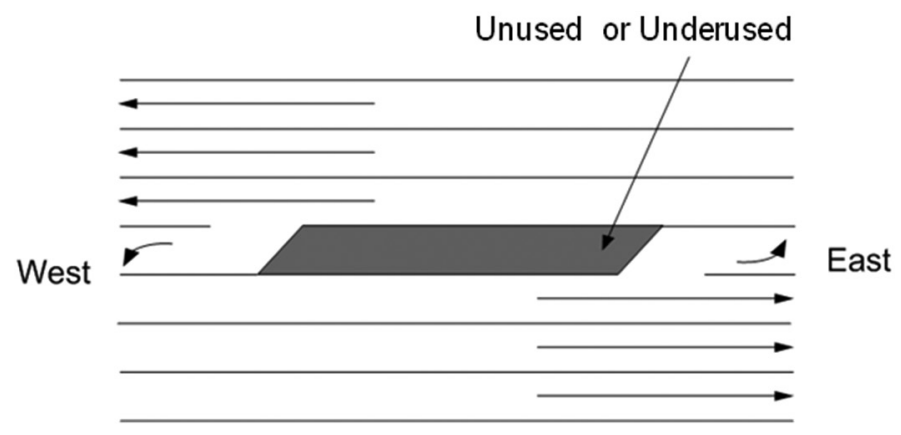

\section{Figure 1. Unused or underused median space on typical arterial section provided with one left-turn lane on each of two ends}

All geometric designs sketched in this paper are used for illustration and comparison. For ease of comparison, the traffic moves along the east-west direction, i.e., horizontally between the left- and right-hand sides of the diagram, in all the sketches. For ease of discussion, the width of right-of-way is measured in the unit of a traffic lane, regardless of whether the traffic lane is a through lane for regular traffic, a left-turn lane, or a dedicated bus lane. Moreover, a passenger platform is treated as being as wide as a traffic lane, regardless of whether it is dedicated to use by only passengers heading in one direction or is shared between passengers heading in either of the two directions. We ignore possible curbside parking altogether in the diagrams. We refer to the portion of a street delimited by two adjacent intersections as a section. For ease of discussion, we refer to a section in which a bus stop is provided as a bus-stop section and refer to a section not provided with a bus stop as a non-bus-stop section.

\section{Efficient, Slanted Geometric Design for Non-bus-stop Section Capitalizing on Unused Median}

The dedicated lanes of the prevailing geometric design for a non-bus-stop section are straight and are perfectly parallel to the longitudinal orientation of the arterial, as illustrated in Figure 2. This configuration provides two general-purpose lanes, one left-turn lane, and one dedicated BRT lane for each direction and requires right-of-way whose width spans eight traffic lanes. This may be a simple and obvious option and may be aesthetic, but it requires more space than necessary. This "straight" configuration and its variations are also the standard configurations 
throughout the VTA light rail system operated by Santa Clara County in California, for sections without a passenger platform. The efficient right-of-way allocation proposed in this paper provides, for this particular example, an identical set of traffic lanes but requires right-of-way that is only seven-lane wide, as illustrated in Figure 3.

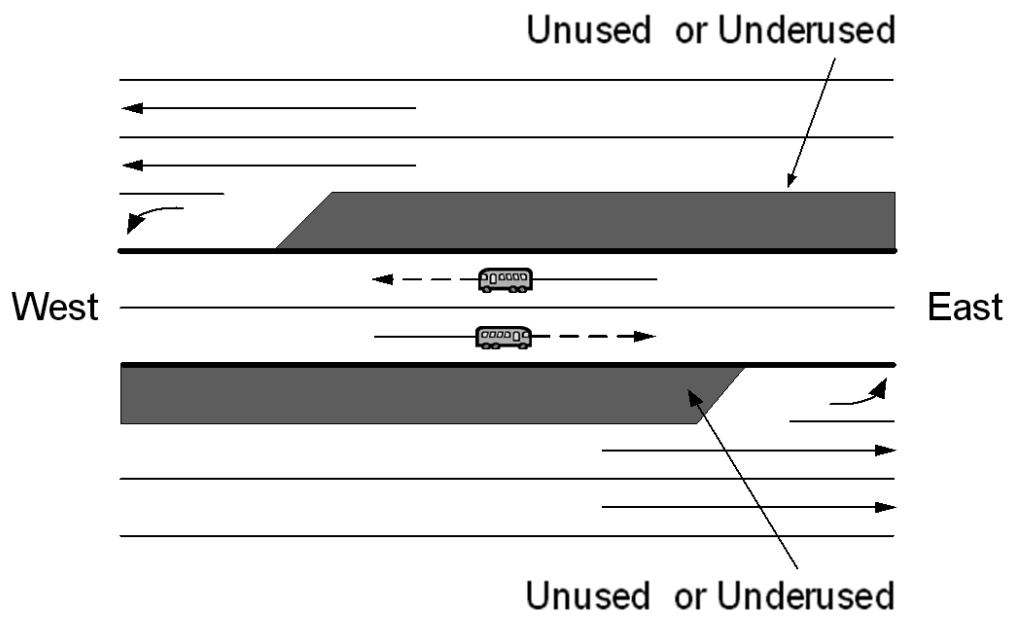

Figure 2. Straight but inefficient geometric design for "non-bus-stop" section of two-dedicated-lane BRT on eight-lane right-of-way: Accommodating two general-purpose through lanes and one left-turn lane for each direction, creating even more unused or underused median space

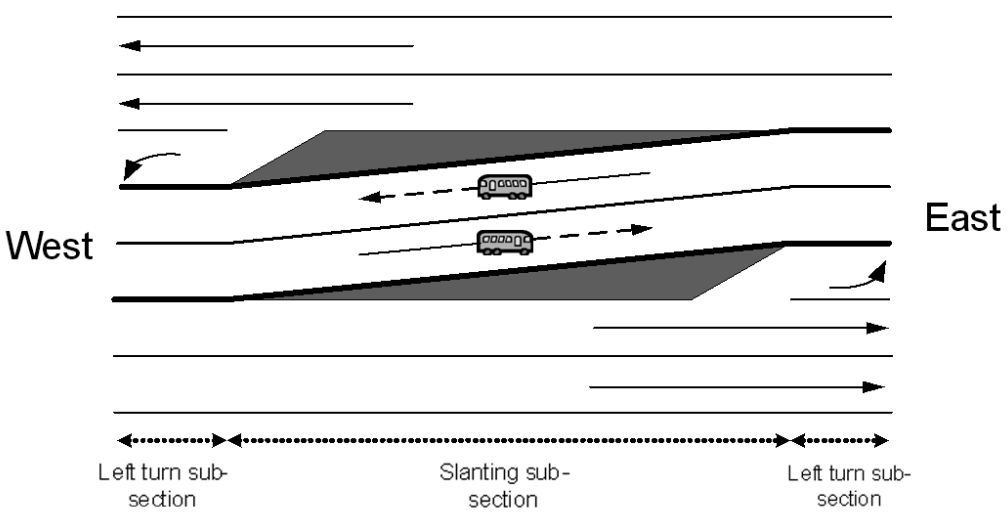

Figure 3. Slanting of dedicated lanes of two-dedicated-lane BRT system and saving of one lane on seven-lane right-of-way for a non-bus-stop section: Accommodating two through lanes and one left-turn lane for each direction 
If we allow part of the dedicated lanes to be slanted with respect to the longitudinal orientation of the arterial, then we can use the otherwise unused or underused median space and, hence, save one lane. We illustrate this idea of slanting by first examining the configuration sketched in Figure 3 for a non-bus-stop section. With slight slanting of the dedicated lanes, the space requirement can be reduced by one traffic lane, from eight (of Figure 2) to seven (of Figure 3) in this particular example.

Often, acquiring additional right-of-way along a busy corridor is infeasible, and dedicated BRT lanes can only be provided through conversion of general-purpose lanes. In such cases, the proposed slanting of BRT lanes can reduce the impact of such conversion on the general traffic to the minimum. It incurs conversion of only two general-purpose lanes. For example, the configuration of Figure 3 can be converted from a roadway of the same width that accommodates three through lanes and one left-turn lane in each direction, as illustrated in Figure 1. This conversion, however, does not allow mid-block left turns for convenient access to locations on the other side of the roadway.

In cases where the available right-of-way cannot accommodate two dedicated BRT lanes but can accommodate one, transit agencies can resort to the operational concept of dynamically reversible one-dedicated-lane BRT system proposed in Tsao et al. (2009a, 2009b, 2009c, and 2010). In that concept, the unused median space can be used for buses traveling in opposite directions to cross each other.

\section{Efficient, Slanted Geometric Design for Bus-stop Section with Passenger Platforms Converted from Selected Left-turn Lanes}

In this section, we first discuss a common geometric design for a bus-stop section of a two-dedicated-lane BRT system and propose an efficient configuration that saves one lane. Although multiple designs for a bus-stop section exist, the required amount of right-of-way is similar. Figure 4 illustrates such a design (AC Transit 2012b). Note that the only difference between this configuration and the one shown in Figure 2 (for a non-bus-stop section) is that parts of the unused median space of the latter are used for passenger platforms. The BRT of this configuration occupies three to four lanes, and three through lanes are taken away from generalpurpose traffic. Although the middle portion of the dedicated space spans four lanes, the portions of the dedicated space located on the two opposite ends of the section occupy only three lanes each. Therefore, this configuration takes away three lanes, not four, for the dedication. 


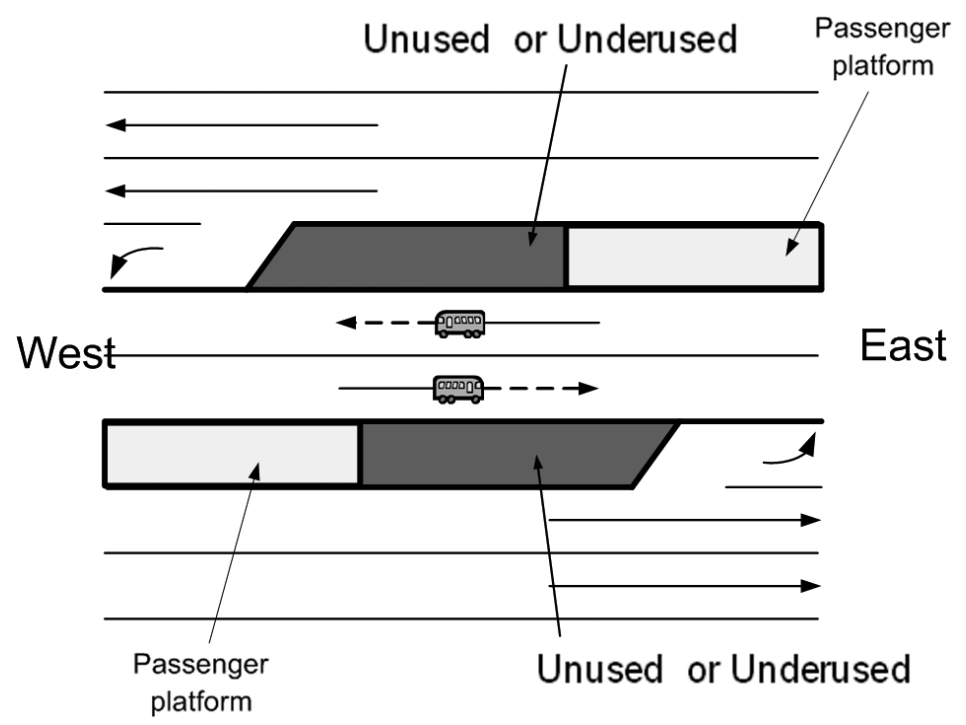

Figure 4. Straight but inefficient geometric design for "bus-stop" section of two-dedicated-lane BRT on eight-lane right-of-way, occupying four lanes, taking away three lanes and accommodating two through lanes and one left-turn lane for each direction

If the left-turn lanes can be sacrificed, the system illustrated in Figure 4 can be improved so that one lane can be saved. Such an improved design is illustrated in Figure 5. Note that the two left-turn lanes on this section, one in each direction, are converted to BRT passenger platforms, and the two dedicated lanes are slanted with respect to the longitudinal orientation of the roadway. Like the configuration illustrated in Figure 4, the two passenger platforms are located on two opposite ends, i.e., east and west, of the section. However, each of the two platforms is located on the opposite end of the section with respect to its counterpart shown in Figure 4. Note that this configuration differs from the one illustrated in Figure 3 (for a non-bus-section) in that the left-turn lanes of Figure 3 are replaced with the two corresponding passenger platforms. 


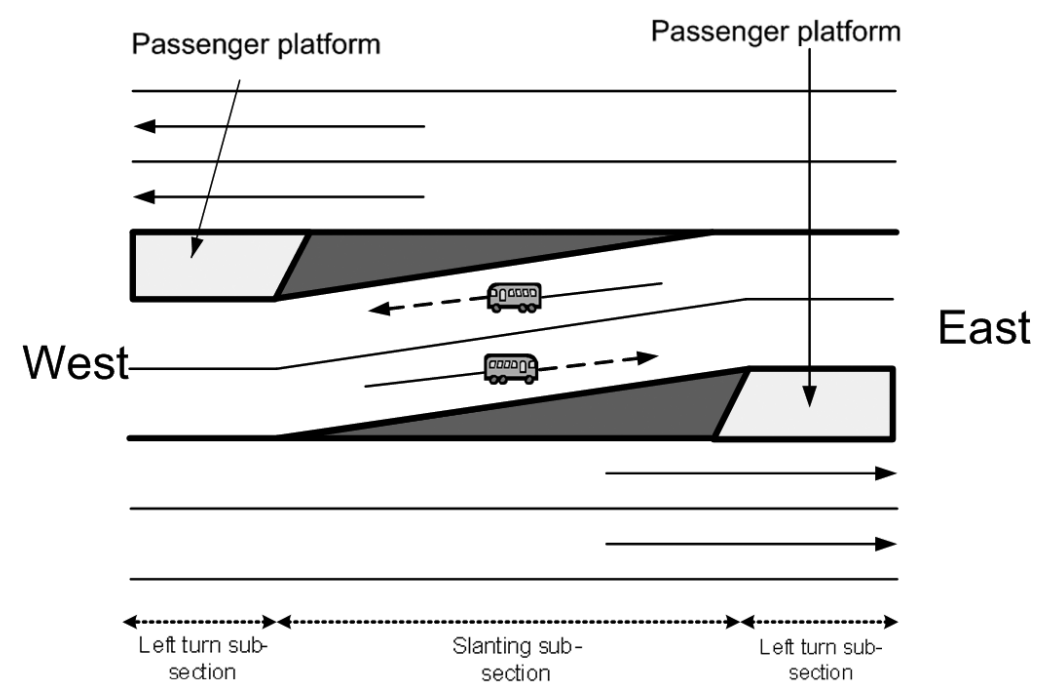

Figure 5. Slanting of dedicated lanes of two-dedicated-lane BRT system and saving of one lane on seven-lane right-of-way for a bus-stop section, sacrificing left-turn lanes for passenger platforms, occupying only three lanes, taking away only two lanes and accommodating two through lanes and one left-turn lane for each direction

A variant of the geometric design of Figure 4 is illustrated in Figure 6 , and it is perhaps the prevailing geometric design. Although different, the two configurations occupy the same amount of space. In fact, Figure 6 can be thought of being formed by "cutting" the eastern half of the bus-stop section of Figure 4 and "pasting" it to the west of the intersection bordering the western half of the same bus-stop section. This design has the advantage of both platforms being located at the same intersection.

Similarly, this configuration can be improved to save one lane. An alternative design is illustrated in Figure 7. In this alternative, two passenger platforms are located on two sides of an intersection. This alternative configuration may have an advantage in that the passenger activities of this bus stop are concentrated at one intersection. Like their conventional counterparts, Figure 7 can be thought of being formed by "cutting" the eastern half of the bus-stop section of Figure 5 and "pasting" it to the west of the intersection bordering the western half of the same bus-stop section. 


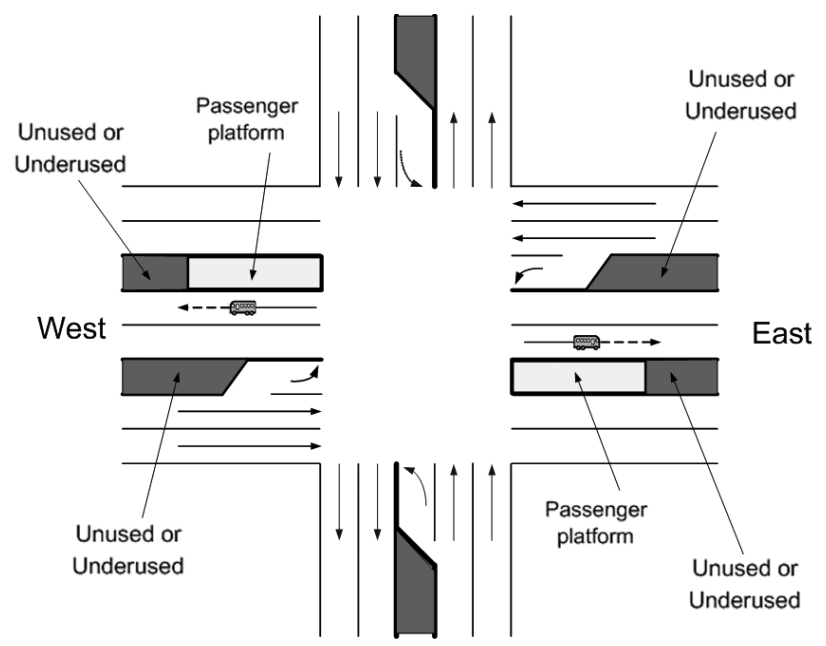

Figure 6. Prevailing, straight but inefficient geometric design for "bus-stop" section of two-dedicated-lane BRT on eight-lane right-of-way occupying four lanes, taking away three lanes and accommodating two through lanes and one left-turn lane for each direction;

platforms at one interaction

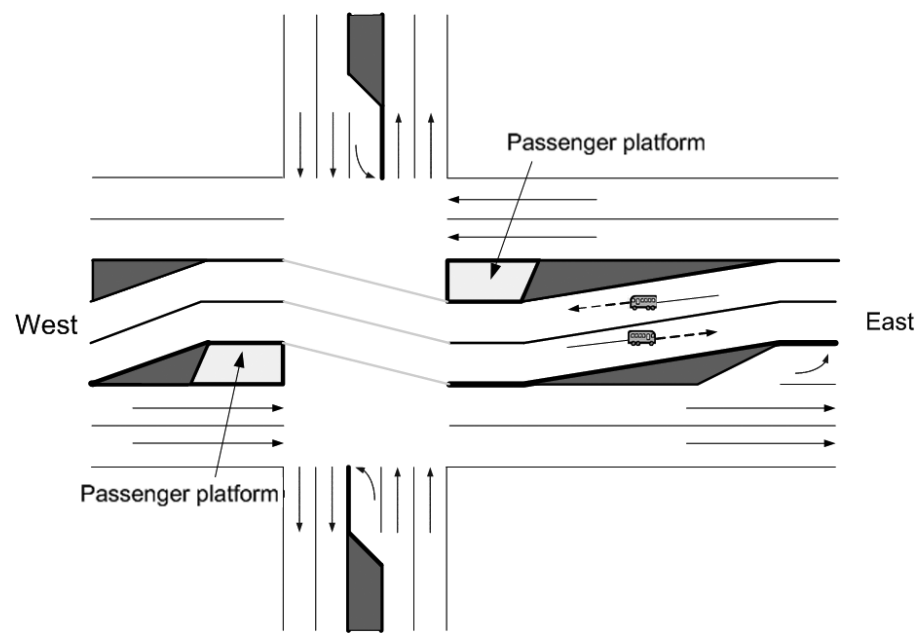

Figure 7. Slanting of dedicated lanes of two-dedicated-lane BRT system and saving of one lane on seven-lane right-of-way for a bus-stop section, sacrificing left-turn lanes for passenger platforms, occupying only three lanes, taking away only two lanes and accommodating two through lanes and one left-turn lane for each direction; platforms at one intersection 


\section{Efficient Geometric Design for Bus-stop Section with Passenger Platform Converted from Unused Median}

Suppose that the left-turn lanes of the configuration illustrated in Figure 5 are not to be sacrificed. Then, the passenger activities can be accommodated in the middle of the section to fully use the unused or underused median space, as illustrated in Figure 8. Note that the platform can be accessed via mid-block crosswalks. However, additional traffic signals will be required for safety, and impact on traffic may be significant. Pedestrian safety may also be an issue because drivers may not be used to such mid-block crosswalks and the companion signals. To enable passenger boarding and alighting, buses must also be equipped with doors on the left-hand side.

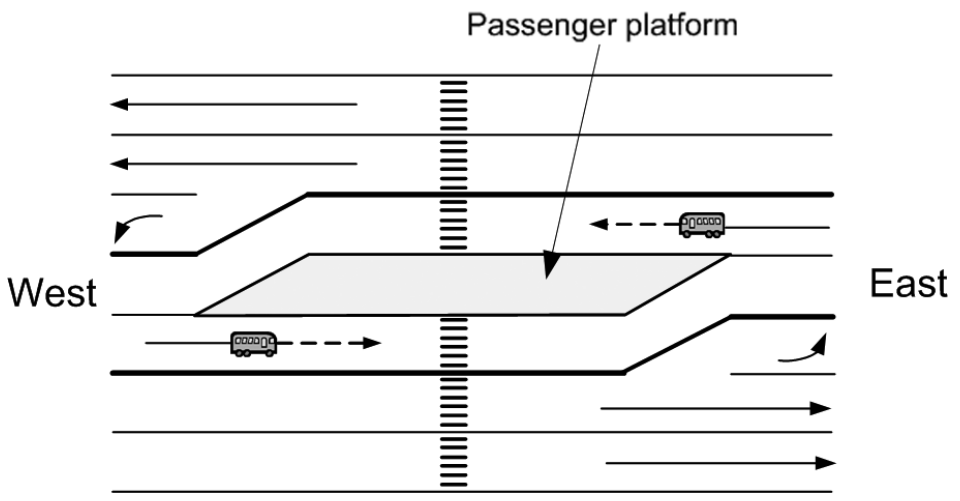

Figure 8. Two-dedicated-lane BRT system taking away two lanes in bus-stop section without sacrifice of left-turn lanes but with bus stop accommodated completely on unused median space

\section{Conclusions}

In merging economies or urban or suburban areas of developed nations where bus transit is already popular, faster and more reliable bus service would be considered "rapid" and may suffice for public support. However, in the U.S., where automobile is the primary mode of personal transportation and only (heavy) commuter rail transit systems, e.g., Bay Area Rapid Transit (BART) system of the San Francisco Bay Area, New York City Subway, etc., have been considered as "rapid" by the general public, their expectation on the speed of a bus rapid transit system may be much higher. This higher speed expectation may only be achievable with a dedicated median busway and TSP, and, hence, the concomitant necessity of efficient space dedication is critical. 
For many current busy commute corridors, a significant part of the street median is underused or unused for traffic purposes. We capitalized on this phenomenon and proposed geometric configurations that more fully use the street median. The efficiency gain is achieved independently and additively through such fuller use for individual sections. Therefore, the proposed configurations offer the highest efficiency-gain potential if the corridor consists of long sections and is equipped with a left-turn lane at each end of each of its sections. In such cases, as long as the street right-of-way is seven-lane wide, a BRT with two dedicated lanes should be geometrically feasible, leaving the remaining five lanes to accommodate two through lanes and one left-turn lane for each direction.

The benefit of the proposed configurations hinges upon two important factors: (1) the prevalence of left-turn lanes along busy commute corridors and (2) the minimum length requirement for a section to accommodate the slanting. We studied two corridors well known to San Jose, California, residents but focused on a nondowntown portion for each. We found an overwhelming presence of left-turn lanes on both. We also derived the minimum length of the slanting portion of the two dedicated lanes as a function of the design speed, superelevation, coefficient of side friction, and lane width. Our study of the geometric configurations of the two non-downtown portions reveals a large amount of unused median (approximately $58 \%$ ) and a high likelihood of section-length sufficiency for accommodating the required slanting. Due to the page limit, these findings will be reported separately. If the right-of-way required for any of the configurations proposed in this paper is not available, then the concept of one-dedicated-lane dynamically-reversible BRT we proposed in an earlier paper may offer a solution (Tsao et al. 2009a, 2009b, 2009c, 2010). When sufficient right-of-way or public support becomes available for a two-dedicated-lane BRT system after implementation of a one-dedicated-lane system, the one-dedicated-lane system can be expanded with ease to two dedicated lanes (Tsao et al. 2009a, 2010). Further efficiency of right-of-way utilization can be achieved for a BRT system (with either one or two dedicated lanes) with the advanced technologies of automated lateral control (Tsao 1998; Al-Kadri et al. 1998).

In addition to the prevailing concepts of a two-dedicated-lane BRT system, many BRT concepts have been proposed or implemented for operations in mixed traffic. Together with these concepts, the configurations and operational concepts we proposed in this paper and those we proposed earlier for a one-dedicated-lane system hopefully constitute a more complete spectrum of implementation options, 
at least from the view point of dedicating right-of-way along a street median. Implementation of any surface transportation system tends to be site-specific; a particular BRT implementation may involve several or even all of these options.

\section{Acknowledgements}

The authors would like to thank California Department of Transportation (Caltrans) and California Partners for Advanced Transit and Highways (PATH) for funding this research under the category of New and Innovative Research Idea (Contract No. 65A0268-6197). They are grateful for the guidance and support generously provided by Mr. Weibin Zhang of PATH and Ms. Sonja Sun and Mr. Bradley Mizuno of Caltrans. Any opinions expressed herein do not necessarily reflect those of Caltrans or PATH. Valuable comments made by two anonymous reviews of the original version of this manuscript are gratefully acknowledged. Some of the configurations proposed in this paper were conceived in an earlier project about (dynamically reversible) one-dedicated-lane BRT funded by Mineta Transportation Institute of the San Jose State University.

\section{References}

AC Transit. 2012a. AC Transit East Bay Bus Rapid Transit Project in Alameda County-Final Environmental Impact Statement/Final Environmental Impact Report (FEIS/FEIR). Alameda-Contra Costa Transit District, Oakland, California. http://www.actransit.org/final-environmental-impact-statementfinalenvironmental-impact-report-feisfeir/. Accessed online December 29, 2012.

AC Transit. 2012b. East Bay Bus Rapid Transit Project-Final Environmental Impact Statement/Report: Community Meetings, February-March 2012. AlamedaContra Costa Transit District, Oakland, California. http://www.actransit.org/ wp-content/uploads/BRT-Exhibit-Boards.pdf. Accessed online on December 29, 2012.

Al-Kadri, M., H. Benouar, and H.-S. J. Tsao. 1998. Intermediate automation concepts for evolution toward automated highway systems. Transportation Research Record 1651.

Callaghan, L., and W. Vincent. 2007. Preliminary evaluation of Metro Orange Line Bus Rapid Transit project. Transportation Research Record 2034: 37-44. 
Carey, G. 2006. Bus Rapid Transit: The Eugene-Springfield, OR, experience. ITE Journal, July: 20-23.

Diaz, R. B., M. Chang, G. Darido, E. Kim, D. Schneck, M. Hardy, J. Bunch, M. Baltes, D. Hinebaugh, L. Wnuk, F. Silver, and S. Zimmerman. 2004. Characteristics of Bus Rapid Transit for Decision Making. Office of Research, Demonstration and Innovation, Federal Transit Administration, U.S. Department of Transportation.

Institute for Transportation and Development Policy (ITDP). 2009. Bus Rapid Transit Planning Guide. New York, April 26. http://www.itdp.org/library/publications/brt-planning-guide-complete. Accessed online on December 29, 2012.

Jarzab, J. T., J. Lightbody, and E. Maeda. 2002. Characteristics of bus rapid transit projects: An overview. Journal of Public Transportation 5(2): 31-46.

Kittelson \& Associates, Inc., et al. 2007. Transit Cooperative Research Program (TCRP) Report 118: Bus Rapid Transit Practitioner's Guide. Transportation Research Board, Washington, DC. http://onlinepubs.trb.org/onlinepubs/tcrp/ tcrp_rpt_118.pdf. Accessed online on December 29, 2012.

Lane Transit District. 2002. Eugene-Springfield, Oregon East-West Pilot BRT. Eugene, Oregon. http://gulliver.trb.org/publications/tcrp/tcrp90v1_cs/Eugene. pdf. Accessed on December 29, 2012.

Levinson, H. S., S. Zimmerman, J. Clinger, and C. S. Rutherford. 2002. Bus rapid transit: An overview. Journal of Public Transportation 5(2): 1-30.

Li, J.-Q., M. K. Song, L. M. Song, W.-B. Zhang, and M. Miller. 2009. Evaluation of cost-effective planning and design options for bus rapid transit in dedicated bus lanes. PATH Research Report UCB-ITS-PRR-2009-14, Institute of Transportation Studies, University of California at Berkeley.

Miller, M. A., C.,-W. Tan, A. Golub, M. Hickman, P. Lau, and W. -B. Zhang. 2006. Development of deployment strategy for an integrated BRT system. PATH Research Report, UCB-ITS-PRR-2006-9, Institute of Transportation Studies, University of California, Berkeley.

Tsao, H.-S. J. 1998. An axiomatic approach to developing partial automation concepts for deployment of automated highway systems and partial invocation of vision-based lane-keeping and adaptive cruise control. Transportation Research Record 1651. 
Tsao, H.-S. J., Y. Dessouky, K. Ingham, R. Ongkowidjojo, and J. R. Tsao. 2010. Developing operating rules and simulating performance for one-dedicated-lane bus rapid transit/light rail systems. PATH Research Report UCB-ITS-PRR-2010-01, Institute of Transportation Studies, University of California at Berkeley, March.

Tsao, H.-S. J., W. Wei, and A. Pratama. 2009a. Feasibility of one-dedicated-lane bus rapid transit/light-rail systems and their expansion to two-dedicated-lane systems: A focus on geometric configuration and performance planning. MTI Report No. 08-01, Mineta Transportation Institute, San Jose State University, San Jose, California. http://www.transweb.sjsu.edu/MTIportal/research/publications/documents/BRT.pdf. Accessed online on December 29, 2012.

Tsao, H.-S. J., W. Wei, and A. Pratama. 2009b. Operational feasibility of onededicated-lane bus rapid transit/light-rail systems. Transportation Planning and Technology 32(3): 239-260. Also translated into Chinese and published in Urban Transport of China 8(2), March: 80-93.

Tsao, H.-S. J., W. Wei, A. Pratama, and J. R. Tsao. 2009c. Launching bus rapid transit with only one dedicated lane for two-way bus traffic on congested corridors. Presented at the 2nd Annual Conference of Indian Subcontinent Decision Science Institute (ISDSI 2009), January 3-5, 2009, Mumbai, India; published in Proceedings: 113-125.

York Region Rapid Transit Corporation. 2012. VivaNext. http://www.vivanext.com/ rapidways. Accessed on December 29, 2012.

\section{About the Authors}

H.-S. JACOB TSAO (jacob.tsao@sjsu.edu) holds a Ph.D. in Operations Research from University of California at Berkeley and is a Professor in the Department of Industrial and Systems Engineering of San Jose State University (SJSU). He is also a Research Associate at the Mineta Transportation Institute of SJSU. Prior to joining SJSU in 1999, he worked at the Institute of Transportation Studies at University of California, Berkeley for seven years and at the AT\&T Bell Laboratories for four years. Details can be found at http://www.engr.sjsu.edu/jtsao.

Agus Pratama (pratamaagus@gmail.com) holds an M.S. in Industrial and Systems Engineering and an M.S. in Software Engineering, both from San Jose State University. He is currently working as a Software Engineer at Workday Inc. 